\title{
Determinantes de la participación laboral en el municipio de San José de Cúcuta y su área metropolitana durante el tercer trimestre del año 2011*
}

Determinants of labor participation in the municipality of San José de Cucuta and its metropolitan area during the third quarter of $2011^{*}$

Determinantes da participação do trabalho no município de São José de Cúcuta e sua região metropolitana durante o terceiro trimestre de $2011^{*}$

\author{
Jessica Dayana Ramírez López ** \\ Luis Miguel López Celemín *** \\ Mario De Jesús Zambrano Miranda **** \\ Universidad Libre de Colombia - Cúcuta
}

Fecha de Recibido: Febrero 02 del 2015

Fecha de Aceptación: Abril 13 de 2015

Fecha de Publicación: Diciembre 15 de 2015

DOI: http://dx.doi.org/10.22335/rlct.v6i3.671

* Articulo producto del proyecto de investigación: Diagnóstico del Mercado de Trabajo y Contexto Socioeconómico de Cúcuta y Área Metropolitana, Universidad Libre Cúcuta.

** Economista, Especialista en Gerencia Financiera, candidata a Magister en Gerencia y Práctica del Desarrollo de la Universidad de los Andes, Gerente Empresarios por la educación Capitulo Norte de Santander. gerentecapntesantander@fundacionex.org.co

*** Economista, Especialista en Gerencia Financiera, Especialista en Contratación Estatal y Especialista en seguridad Social: miguel.celemin@unilibre.edu.co

**** Economista, Lic Ciencias Sociales, Especialista Gestión Pública, Magister en Gobierno y Política Pública. Líder del grupo de investigación Competitividad y Sostenibilidad para el Desarrollo de la Universidad Libre. mariod.zambranom@unilibre.edu.co

\section{Resumen}

El presente trabajo busca determinar la influencia de las condiciones socioeconómicas del hogar en la participación laboral bajo el modelo OCIO CONSUMO, para el municipio de san José de Cúcuta y su área metropolitana durante el tercer 
trimestre del año 2011. El análisis se hará desde la perspectiva de Gary Becker; los resultados muestran que las variables sexo, tasa de desempleo del hogar y jefatura del hogar, son las que mayor efecto positivo tienen en la probabilidad de participar en el mercado laboral y las variables ingresos laborales, edad y pareja las que menor efecto presentan.

Palabras Claves: Ocio, Consumo, mercado laboral, participación laboral.

\section{Abstract}

This work seeks to determine the influence socioeconomic conditions at homes on labor participation according to leisure-consumption model, in San José de Cucuta and its metropolitan area during the third quarter of 2011. This analysis will be done from the Gary Becker's perspective; the results show that the variables sex, home unemployment rate and head of homes, are those that have the greatest positive effect on the probability of participating in the labor market and the variables labor income, age and couple have the least effect.

Keywords: Leisure, Consumption, labor supply, labor demand

\section{Resumo}

Este trabalho procura determinar a influência das condições socioeconômicas nos domicílios, tendo como pressuposto a participação do trabalho segundo o modelo lazer-consumo, em São José de Cúcuta e região metropolitana, no terceiro trimestre de 2011. Essa análise será feita a partir da perspectiva do Gary Becker. Nesse sentido, os resultados demonstram que as variáveis sexo, taxa de desocupação domiciliar e chefe de domicílio são as que têm maior efeito positivo sobre a probabilidade de participação no mercado de trabalho. Por outro lado, as variáveis rendam do trabalho, idade e casal têm o menor efeito
Palavras-chave: Consumo, oferta do trabalho, demanda do trabalho

\section{Introducción}

En el análisis neoclásico convencional del mercado laboral, se parte de la importancia de las condiciones de la oferta, que determinan las decisiones de participar o no del individuo. La conducta racional de este individuo está relacionada con el objetivo de buscar maximizar su utilidad, donde se enfrenta a una disyuntiva entre el ocio y el consumo, sujeto a una restricción de ingreso. Por su parte, Becker (1965) incluye un enfoque modificado de la teoría del consumidor, donde incorpora la importancia de la distribución del tiempo; es decir, diferencia el tiempo dedicado al trabajo salarial y el tiempo dedicado a las actividades del hogar, lo que conlleva a pensar el hogar como una pequeña firma. La anterior línea la desarrollan Castellar \& Uribe (2001) de donde parte el presente artículo y que tiene como objetivo determinar la influencia de las condiciones socioeconómicas del hogar en la participación laboral bajo el modelo Ocio - Consumo para el municipio de san José de Cúcuta y su Área Metropolitana durante el tercer trimestre del año 2011.

Aunque se han presentado estudios sobre el tema como los desarrollados por Ribero \& Meza (1997), Santamaría \& Rojas Delgadillo (2001), Posada \& Arango (2002); Posada, Arango, \& Charry (2006) a nivel nacional y los de Castellar \& Uribe (2001) y Aldana \& Arango (2007) a nivel regional, en Cúcuta y su Área Metropolitana no se ha realizado análisis sobre la oferta laboral del municipio.

El presente trabajo utiliza un modelo de variable dependiente dicótoma tipo Probit, en donde, la variable regresada toma valores de 1 cuando el individuo participa laboralmente y 0 cuando no participa. Las variables explicativas se determinan por el salario de reserva que incluyen las características socioeconómicas de los individuos y de su entorno (sexo, estado civil, jefatura del hogar, 
ingresos laborales, si está dentro del régimen subsidiado de salud y tasa de desempleo en el hogar) y el salario de mercado que incluye la acumulación de capital humano; en este caso, serán las variables educación y edad (proxy de la experiencia). La información utilizada es suministrada por la gran encuesta integrada de hogares (GEIH) elaborada por el DANE para el tercer trimestre del año 2011. Los resultados muestran que las variables sexo, tasa de desempleo del hogar y jefatura del hogar, son las que mayor efecto positivo tienen en la probabilidad de participar en el mercado laboral y los variables ingresos laborales, edad y pareja las que menor efecto presentan.

El articulo está estructurado de la siguiente manera: primero, se elaborará una breve revisión literaria entorno a los estudios más representativos de la oferta laboral; seguido del marco teórico del modelo Ocio-Consumo; en tercer lugar, un análisis descriptivo del tercer trimestre del 2011; el cuarto apartado es la estimación econométrica bajo un modelo tipo Probit y por último las conclusiones.

\section{Antecedentes}

La participación laboral es un fenómeno estudiado ampliamente en la literatura económica nacional e internacional. Los estudios realizados han sido un gran aporte al debate y comprensión de la realidad socioeconómica de aquellos que ofrecen su fuerza de trabajo, sus análisis con fundamentación microeconómica son el resultado de múltiples metodologías que muestran las condiciones sociales de agentes que participan en el mercado laboral, y además, sirven de base para la elaboración de políticas económicas y sociales frente al tema del empleo.

En la literatura internacional el trabajo de Heckman y Killingsworth, permite señalar la importancia de las diferencias entre hombres y mujeres a la hora de ofrecer su fuerza laboral. En el estudio mediante modelos con datos panel se analizan los determinantes de la participación laboral femenina para el Oeste de los Estados Unidos; y encuentran que el comportamiento de la oferta laboral en las mujeres tiene implicaciones de muchos fenómenos como el matrimonio, la fertilidad, el divorcio, las diferencias salariales entre hombres y mujeres y la existencia de niños menores de seis años en el hogar (Killingsworth \& Heckman, 1986). Un segundo trabajo es el del Doctor Pencavel, el cual resalta las diferencias en la participación laboral de los hombres sobre las mujeres, prestando especial interés en la responsabilidad del hogar de los mismos, allí a través de modelos econométricos encuentra una baja elasticidad en las horas de trabajo, en comparación con el salario y además una tendencia negativa de la participación masculina de los países más desarrollados (Pencavel, 1986).

Dentro los estudios más significativos de participación laboral en los últimos años se encuentra el análisis realizado por el profesor Garavito, C. a la oferta de trabajo del hogar remunerado en el Perú en el periodo comprendido entre 2007 - 2014, en la cual determinó mediante estadística inferencial que hay mayores probabilidades para las mujeres de emplearse en trabajo del hogar remunerado (THR), de igual forma, encontró en los determinantes de la oferta de THR alta inelasticidad entre horas de trabajo tasa salarial. Por último, entre los resultados evidenció que los ingresos no laborales (familiares) actúan como piso de los ingresos laborales y tienen un efecto negativo directo con las horas de trabajo; asimismo, las trabajadoras casadas, en unión de hecho o jefas de hogar trabajan menos horas (Garavito, 2017).

Recientemente son múltiples los artículos de investigación que tiene por objeto de estudio la participación laboral, especialmente en los determinantes que influyen a la hora de ingresar al mundo del trabajo, tal vez uno de los estudios mas rigurosos lo presentó el profesor Tomaselli en un documento publicado por Comisión Económica para América Latina y el Caribe (CEPAL), titulado "Caracterización de la participación laboral en 
Chile". Allí describió los diferentes grupos poblacionales y su impacto dentro de la oferta laboral, en los cuales evidenció en el país de Chile un bajo promedio de la población especialmente joven y de sexo mujer, respecto al latinoamericano.

Dentro de las conclusiones determina que la inactividad económica está fuertemente marcada por el sexo y la edad de las personas. "Las mujeres en todo rango etario se hallan fuera del mercado laboral por razones familiares (cuidado de niños $y$ quehaceres del hogar). Mientras que los hombres jóvenes justifican su inactividad por razones de estudio y en caso de adultos mayores las razones estarían vinculadas con la jubilación.." (Tomaselli, 2017)

En el ámbito nacional los estudios realizados sobre la participación laboral con fundamentación microeconómica son múltiples. El primer trabajo inferencial con variables dicótomas, lo realizó Ribero y Meza, el cual explica mediante ejercicios econométricos tipo Probit la participación laboral de hombres y mujeres durante los años 1976 a 1995, además realiza simulaciones con los cambios observados en algunas variables. De esta manera, encontró que algunas variables, incluidas el nivel educativo, la edad, la jefatura del hogar y la participación laboral del conyugue tienen un impacto positivo en la probabilidad de participar en el mercado laboral en ambos sexos. La asistencia escolar, el número de personas en el hogar y los niños menores de seis años en el hogar presentan un efecto negativo sobre la probabilidad de participar de las mujeres. Adicionalmente, las autoras realizan simulaciones ante cambios en el nivel educativo, la asistencia escolar, el número de personas en el hogar, la jefatura del hogar, los niños menores de seis años en el hogar y los ingresos del cónyuge para observar el comportamiento que se obtendría si se cambiaran los valores medios de algunas variables. Se encontró que el efecto más notorio fue la participación laboral del cónyuge y la educación, puesto que el cambio que se realizó en dichas variables tuvo los mayores impactos en la probabilidad de participar en el mercado laboral (Ribero \& Meza, 1997).

En el nuevo siglo el trabajo de Santamaría y Rojas, permite identificar que el aumento de la participación laboral en las mujeres fue debido a cambios demográficos, institucionales y culturales, en donde el papel de la mujer en la sociedad y el incremento de la educación, tuvieron implicancias en las decisiones de fertilidad y cambios en la normatividad que permitieron una mayor absorción de la mano de obra femenina. Además, se evidenció la existencia de la hipótesis del trabajador adicional; cuando los ingresos del hogar disminuyen se incrementa la participación de algunos de los miembros del hogar especialmente las mujeres y los jóvenes. Los autores utilizaron modelos econométricos tipo Logit durante los años 1983, 1988, 1992, 1995, 1999 y 2000. (Santamaría \& Rojas Delgadillo, 2001)

Los principales resultados que obtuvieron son una mayor probabilidad de participar laboralmente cuando se posee el nivel de educación superior completa, siendo ésta mayor para las mujeres que para los hombres, La presencia de niños menores a seis años disminuye la probabilidad de participar de las mujeres, contrario a lo que ocurre con los hombres, efecto negativo en las mujeres cuando hay presencia de minusválidos en el hogar, mayor probabilidad en las mujeres si son solteras, mientras que en los hombres sucede lo contrario y por último se encuentra que el aumento de los desempleados en el hogar aumenta la probabilidad de participar tanto en las mujeres como en los hombres.

Otro de los trabajos más significativos y pioneros es el realizado por Posada y Arango (2002), donde se presentan nuevos aportes al estudio de los determinantes de la participación laboral, ya que analizan el conjunto de etapas de la Encuesta Nacional de Hogares elaborada por el DANE durante los años 1984 - 2000, que no se había logrado analizar. Los autores realizaron un modelo econométrico tipo Probit para cuatro categorías del 
hogar: mujeres comprometidas (casadas o en unión permanente), mujeres no comprometidas (solteras, viudas o separadas), hombres comprometidos y hombres no comprometidos. En los resultados se encuentra que en la significancia de los coeficientes, las categorías mejor explicadas fueron las mujeres comprometidas y no comprometidas. El modelo fue menos exitoso para los hombres no comprometidos y menos aún para los hombres comprometidos. La tasa de desempleo del hogar, los años de educación y la edad, fueron unas de las variables que más influyeron de forma positiva a la hora de participar laboralmente, tanto en los hombres como en las mujeres no comprometidos; no obstante, la variable de mayor efecto negativo en el modelo fue la riqueza de los hogares. (Posada \& Arango, La participación laboral en Colombia, 2002)

El trabajo de Uribe, Ortiz y Correa analiza la decisión de participar laboralmente mediante tres consideraciones: 1) La participación en la oferta de trabajo, 2) la búsqueda de empleo y 3) la calidad del empleo. En este sentido, se utilizan dos tipos de modelos econométricos. En el primero se hacen ejercicios econométricos de elección binaria para explicar las dos primeras consideraciones; mientras el segundo, se utiliza un modelo de elección multinomial que explica la tercera consideración, en donde 0 significa inactivo, 1 desempleado, 2 formal y 3 informal.

Los autores dejan claro la importancia de la demanda de trabajo para explicar el comportamiento de las decisiones laborales, puesto que consideran que el rango de opciones de los individuos está determinado por la estructura económica que a su vez contiene la estructura de la oferta laboral y la demanda laboral. De lo anterior, arguyen los autores que debido a la insuficiencia de datos sobre demanda laboral en la Gran Encuesta Integrada de Hogares del DANE, no fue posible estimar su incidencia. Los resultados, aunque fueron altamente significativos en los parámetros, mostraron bajos ajustes de bondad, lo que se atribuye a la ausencia del análisis de la demanda laboral. (Uribe, Ortiz, \& Correa, 2006)

A nivel regional los trabajos más conocidos son los realizados por Castellar \& Uribe y el de Aldana \& Arango. Los primeros analizaron los determinantes de la participación en el mercado de trabajo del área metropolitana de Cali en Diciembre de 1998, y por medio de modelos econométricos tipo Logit, Probit y Modelo de Probabilidad Lineal (MPL) se llegó a la conclusión que el modelo Ocio -Consumo es una buena herramienta para explicar la decisión de participación laboral, donde las variables educación y experiencia laboral son la que explican de mejor forma el salario esperado en el mercado, y la posición en el hogar y el sexo la mejor explicación del salario de reserva. (Castellar \& Uribe, 2001)

El segundo trabajo denominado Participación Laboral en Ibagué para el periodo 2001 - 2005, corrobora la hipótesis de la tasa general de participación como determinante fundamental en la alta tasa de desempleo, mediante tres modelos econométricos tipo Probit aplicados a las 13 áreas metropolitanas, a la Ciudad de Ibagué y uno conjunto para la ciudad y las trece áreas metropolitanas. Los determinantes fundamentales son; el nivel educativo, que arroja como resultado una alta probabilidad de participar si se tiene un nivel mayor de educación; la edad, donde la población entre 30 y 35 años tiene una mayor probabilidad de participar que los que se encuentran entre el rango de 42 a 47 años de edad, y el número de desempleados en el hogar que aumenta la participación de la población más joven. (Aldana \& Arango, 2007)

\section{Marco Teórico}

El modelo que sustenta el estudio de la oferta laboral y sus determinantes es el modelo Ocio Consumo. En sentido abstracto se encuentra a un individuo racional que maximizando su función de utilidad debe elegir entre los bienes consumo y ocio, donde se entiende el consumo como las horas 
dedicadas a laborar y el ocio las horas dedicadas a actividades distintas al trabajo.

En la teoría tradicional del consumidor, el individuo maximiza la función de utilidad $U$ de bienes y servicios comprados en el mercado de la siguiente forma:

$$
U: f(X 1, X 2)
$$

Donde $\mathrm{X} 1$ y X2 son los bienes que le proporcionan utilidad y de los cuales debe elegir conforme a sus preferencias. Sujeto a una restricción de ingreso, $\Sigma$ pi $x i=I$; donde $P i$, es el precio del bien $X i$, y l, es su ingreso. La condición de equilibrio se da cuando la utilidad marginal UMi de cada bien es proporcional a su precio.

$$
\partial \mathrm{U} / \partial \mathrm{Xi}=\mathrm{UMi}=\lambda^{\star} \mathrm{Pi} \quad \mathrm{i}=1, \ldots . \mathrm{n}
$$

Donde $\lambda$ es la utilidad marginal del ingreso.

Becker, incluye un enfoque modificado de la teoría del consumidor, donde incorpora la distribución del tiempo; es decir, diferencia el tiempo dedicado al trabajo salarial y el tiempo dedicado a las actividades del hogar, a éste último le otorga vital importancia en el desarrollo de su teoría como lo evidencia en la publicación: A theory of the allocation of the time en The Economic Jornual :

"La asignación y eficiencia del tiempo no trabajado puede ser ahora más importante para el bienestar económico que el tiempo dedicado al trabajo. Sin embargo, es poca la atención prestada por los economistas" (Becker G. , A Theory of the Allocation of Time, 1965)

De ésta forma, comprende el hogar no solo como un grupo de individuos que demandan bienes, sino como una "pequeña fábrica" de producción de bienes que generan utilidad dentro del hogar; por lo tanto, su tiempo de elaboración es fundamental dentro de la función de utilidad, éste aporte es el punto diferenciador entre la tradicional teoría del consumidor y la propuesta de Becker.
El bien producido en el hogar es llamado $Z_{\text {, }}$ siguiendo la función:

$$
Z i=f(X i, T i)
$$

Donde $\mathrm{X}$ son los bienes del mercado y $\mathrm{T}$ es el tiempo que se dedica a producir los bienes.

Por lo tanto, la función de utilidad se combina en tiempo para maximizarla de la siguiente forma:

$$
U: f(Z 1, Z 2, \ldots Z n)
$$

El individuo presenta una restricción presupuestaria dada por:

$\Sigma$ pi $x i=l=V+T w^{*} W$

Donde I es el ingreso total, $\mathrm{V}$ los ingresos no laborales y Tw son las horas gastadas en trabajo y W son los ingresos dados por cada unidad de Tw.

Y una restricción de tiempo:

$\Sigma T i=T h=T-T w$

Donde Th es el tiempo gastado en el hogar y $T$ el tiempo disponible total del individuo.

Ahora se ha dejado claro que las opciones de elección de bienes tienen restricciones de tiempo y presupuesto, del cual se pueden combinar en una sola del total de recursos:

$\Sigma$ pi $\mathrm{xi}+\Sigma \mathrm{Ti} * \mathrm{~W}=\mathrm{V}+\mathrm{T}^{*} \mathrm{~W}=\mathrm{S}$ (6)

Donde $S$ es el ingreso total, potencial o el ingreso monetario cuando todo el tiempo es usado en el mercado. De esta forma, la condición de equilibrio para maximizar la utilidad se da:

$$
U i=\partial U / \partial Z i=\lambda
$$

Donde $\lambda$ es la utilidad marginal del ingreso. 
Por su parte, Castellar y Uribe exponen el modelo Ocio-Consumo, incluyendo la variable tiempo en el análisis de la maximización de la utilidad basándose en la anterior propuesta de Becker, de igual manera se plantea la función de utilidad (Castellar \& Uribe, 2001):

$$
U(C, L)
$$

Donde $C$ es el consumo del individuo y $\mathrm{L}$ el tiempo dedicado al ocio. Así mismo, presenta una restricción presupuestaria de la forma:

$$
C+w L=y+w T
$$

Donde w es la tasa de salario, y es el ingreso no asalariado y $T$ es el tiempo total del agente.

Si L es el tiempo dedicado al ocio del agente, entonces $\mathrm{H}$ es el tiempo dedicado al mercado de trabajo.

$$
H=T-L
$$

El gráfico 1 muestra la situación de restricción del tiempo y el ingreso:

\section{Gráfico 1}

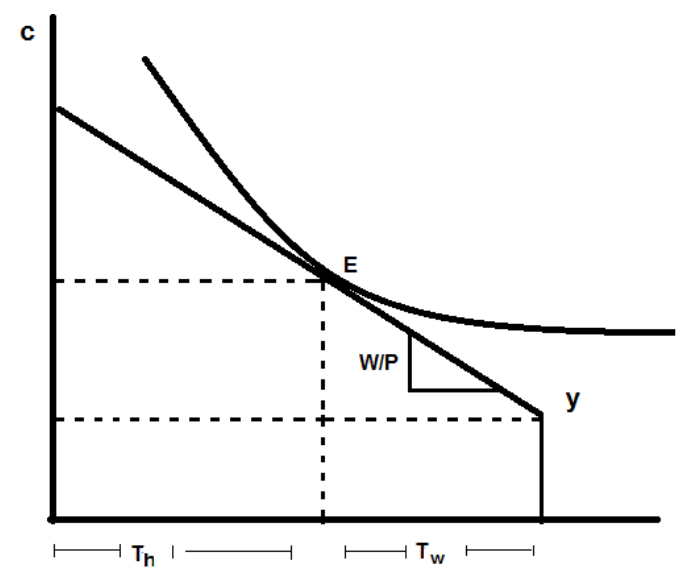

Como se ilustra, la tangente de la restricción presupuestaria es igual a la razón de las utilidades marginales de los bienes, por lo tanto, la condición de equilibro se da cuando la curva de indiferencia se encuentre con la restricción de presupuesto, es decir por la tasa de salario:

U'L<smiles>[13CH]=[W]</smiles>

$U^{\prime} \mathrm{C}$

De esta forma se puede observar que la maximización de la función de utilidad expuesta por Castellar y Uribe se deriva del enfoque modificado de la teoría del consumidor realizada por Becker. No obstante, Castellar y Uribe (ibídem) demuestran la incidencia del salario de reserva en la decisión de participar o no en el mercado laboral, en ese mismo sentido, el estudio realizado por Castiblanco en la población de emprendedores informales determinó de manera similar, una relación negativa entre el incremento del salario de reserva por ingresos familiares y el salario de mercado. (Castiblanco, 2018)

Por lo anterior, es necesario preguntarse que ocurriría si el individuo llegará al límite y dedicará todo su tiempo en Ocio, es decir cuando $L=T$ y $C=Y$. En este caso, la pendiente de la curva de utilidad deberá coincidir con la tasa de salario critica o salario de reserva de la siguiente forma: 


\section{Gráfico 2}

Salario de Reserva

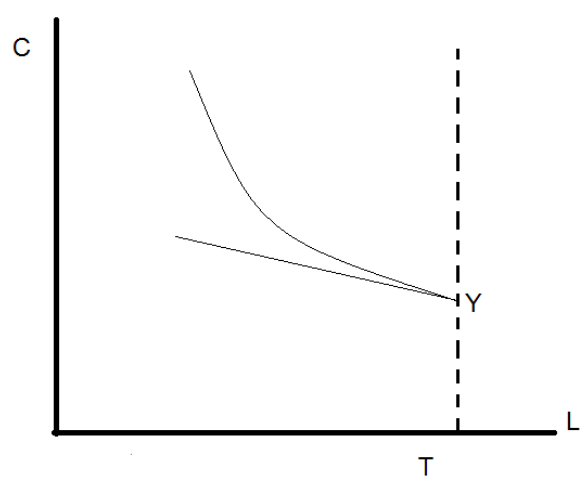

Fuente: Elaboración propia.

El salario de reserva es el precio del tiempo si el agente decide no participar en el mercado de trabajo, también es llamado precio sombra o precio implícito, puesto que es el valor que se deja de percibir cuando se decide no trabajar (Becker, 1965).

Puede darse dos tipos de soluciones: la primera cuando el individuo decide participar (si su salario de reserva es menor que su salario de mercado $\mathrm{W}^{*}<\mathrm{W}$; y otra cuando el individuo decide NO participar (si su salario de reserva es mayor a su salario de mercado $W^{*}>W$ ). Se representa de la siguiente forma en el Gráfico 3:

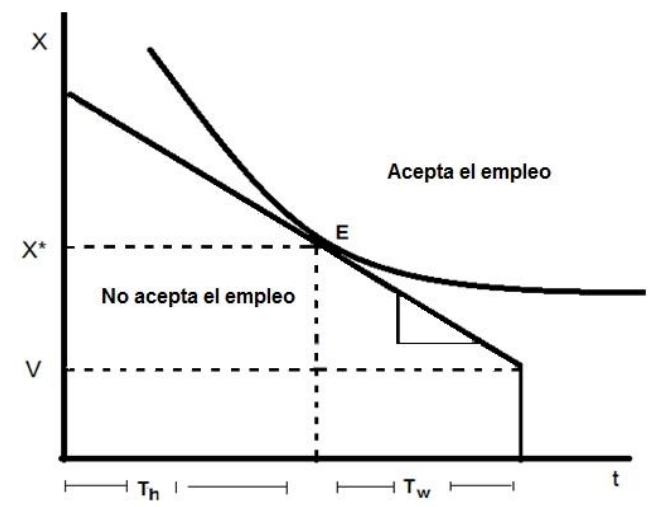

Fuente: Elaboración propia.
Cuando el individuo acepta el empleo los puntos de la isocuanta están por encima de la tangente de la restricción presupuestaria o el salario critico $\mathrm{W}^{*}, \mathrm{y}$ cuando no acepta el empleo, los puntos se encuentran por debajo de la tangente de la restricción, tal como se aprecia en el Gráfico 3.

Es importante rescatar que, aunque la línea teórica de Castellar y Uribe coincide con lo expuesto por Gary Becker hay diferencias conceptuales. Becker en A Theory of the Allocation of Time (1965), Teoria Economica (1977) y A treatise on The Family (1981), no utiliza el término "Ocio", debido a que considera que el tiempo dedicado a las actividades del hogar es "productivo", en el sentido que los individuos son los productores de los bienes Zi. Además, hace parte del ingreso total cuando se deja de percibir dinero por las actividades del hogar.

\section{Análisis descriptivo del tercer trimestre del año 2011}

Las estadísticas del mercado laboral para el Municipio de San José de Cúcuta en el tercer trimestre del año 2011, según los micro datos anonimizados de la Gran Encuesta Integrada de Hogares (GEIH), reflejan una distribución de la población por sexo es de $53 \%$ para las mujeres y $47 \%$ para los hombres, evidenciando un $6 \%$ de superioridad en la participación femenina. El nivel educativo de la población total encuestada muestra que el $3,17 \%$ posee preescolar, el $30 \%$ posee básica primaria, seguido por básica secundaria con un porcentaje del $21,32 \%$, la educación media con $17,62 \%$ y por último la educación superior universitaria con un $15,41 \%$.

En cuanto a la participación según el estado civil, se encuentra que el 32,09\% está soltero, el 17,30\% convive con su pareja en unión libre hace más de dos años, el 17,61\% está casado, el 9,53\% está divorciado o separado y por último un 3,71\% esta viudo.

En lo que respecta a la seguridad social en salud, el $43,97 \%$ se encuentra dentro del régimen 
$Y=\beta 0+\beta 1 X 1+\beta 2 X 2+\beta 3 X 3+\beta 4 X 4+\beta 5 X 5+\beta 6 X 6$

$+\beta 7 X 7+\beta 8 X 8+\varepsilon i$

régimen contributivo, el 5,19\% está dentro de régimen especial en salud y por último un 16,59\% no posee ningún tipo de seguridad social en salud. Las anteriores cifras reflejan una cobertura muy amplia en los subsidios de salud y una cifra alarmante de población desprotegida en seguridad social, resultado del ausentismo del empleo formal.

La posición en el hogar se encuentra representada por hijos e hijastros con un porcentaje de $40,63 \%$, seguido por un $26,63 \%$ de participación de jefes de hogar y un $15,62 \%$ del conyugue o pareja. Las demás categorías no cuentan con la misma representatividad en la participación; sin embargo, cabe resaltar que el servicio doméstico está representado por un $0.23 \%$.

Los ingresos laborales y no laborales por nivel educativo reflejan que tanto las personas que no poseen ningún nivel educativo y los que poseen primaria tienen en promedio ingresos laborales superiores a los no laborales; a partir de ahí la relación cambia, los que poseen nivel de básica secundaria, media y universitarios reciben ingresos superiores no laborales explicados en parte por lo que

\section{Estimación de los determinantes de la participación laboral}

La verificación empírica del modelo Ocio-Consumo se realiza a través de un modelo de variable dependiente dicótoma tipo Probit, en donde, la variable regresada toma valores de 1 cuando el individuo participa laboralmente y 0 cuando no participa. Las variables explicativas se determinan por el salario de reserva que incluyen las características socioeconómicas de los individuos y de su entorno, y el salario de mercado que incluye la acumulación de capital humano estudiado por Becker (1964). En este caso, serán las variables educación y edad (proxy de la experiencia) las utilizadas. La especificación del modelo se da de la siguiente forma:
Donde, las variables explicativas corresponden a Sexo ( 1 es hombre y 0 mujer), Edad (variable proxy de la experiencia), Nivel educativo (último título alcanzado), Posición en el hogar ( 1 si es Jefe de hogar y 0 si no), Estado civil (1 si tiene pareja y 0 si no), Seguridad Social en salud ( 1 sí pertenece al Régimen Subsidiado y 0 si no), Ingresos laborales y Tasa de desempleo del hogar.

De esta manera, se presentan los resultados de la estimación de los determinantes de la participación laboral en el municipio de San José de Cúcuta y se Área Metropolitana:

\begin{tabular}{|c|c|l|}
\hline $\begin{array}{c}\text { VARIABLE } \\
\text { DEPENDIENTE }\end{array}$ & $\begin{array}{c}\text { PARTICIPA EN EL } \\
\text { MERCADO } \\
\text { LABORAL }\end{array}$ \\
\hline $\begin{array}{c}\text { VARIABLES } \\
\text { INDEPENDIENTES }\end{array}$ & \begin{tabular}{c} 
PROBIT \\
\cline { 2 - 3 }
\end{tabular} & $\begin{array}{l}\text { Cofror } \\
\text { Estan. }\end{array}$ \\
\hline Intercepto & $-1,95552^{*}$ & 0.079515 \\
\hline Sexo & $1,062019^{*}$ & 0.052656 \\
\hline Pareja & $0.095964^{* *}$ & 0.051388 \\
\hline Tasa de Desempleo del & $0.447633^{*}$ & 0.089585 \\
\hline Hogar & & \\
\hline Jefe de Hogar & $0.243779^{*}$ & 0.062375 \\
\hline Nivel Educativo & $0.165865^{*}$ & 0.022749 \\
\hline Régimen Subsidiado & $0.175514^{*}$ & 0.049853 \\
\hline Edad & $0.019121^{*}$ & 0.001424 \\
\hline Ingresos Laborales & $4.30 \mathrm{E}-06^{*}$ & $1.46 \mathrm{E}-07$ \\
\hline Numero de Observaciones & \multicolumn{2}{|c|}{5104} \\
\hline Prueba de Significancia & Wald chi2 $=1389.281$ \\
Global & Prob >chi2 $=0.000$ \\
\hline Pseudo R2 & \multicolumn{2}{|c|}{0.476401} \\
\hline RV & 3276.518 \\
\hline AIC & \multicolumn{3}{|c|}{0.709078} \\
\hline
\end{tabular}

Fuente: DANE, Elaboración propia. *Nivel de Significancia del $1 \%,{ }^{* *}$ Nivel de Significancia del $5 \%$.

En donde se aprecia, un buen ajuste conjunto debido a los resultados arrojados por la prueba de significancia de Wald y la Razón de Verosimilitud; adicionalmente, el Pseudo R2 y el criterio Akaike info criterion (AIC) presentan buenos resultados para la evaluación del modelo. En cuanto a la significancia de las variables, se obtiene que todas las variables a excepción de la variable pareja, presentan un nivel de significancia del $1 \%$; es decir, 
las variables estudiadas si aportan información relevante al modelo en forma individual. Los signos que presenta la estimación en las variables explicativas son positivos a excepción del intercepto, indicando que las condiciones socioeconómicas del individuo y de su entorno presentan impactos positivos en la decisión de participar en el mercado laboral; por otro lado, el signo negativo en el intercepto indica que el municipio es poco propenso a participar laboralmente.

Con el fin de obtener mejores resultados, se elaboró la predicción de las probabilidades para determinar el efecto marginal de cada una de las variables explicativas y los cambios en la probabilidad de la variable regresada. De esta manera, se obtuvo un aumento en la probabilidad de participar laboralmente de todas las variables utilizadas (Tabla 2)

Tabla $N^{\circ} 2$ Cálculo de probabilidades para la población masculina:

\begin{tabular}{|l|l|}
\hline \multicolumn{1}{|c|}{ VARIABLE } & PROBABILIDAD \\
\hline SEXO & $21.5526 \%$ \\
\hline EDAD & $0.3880 \%$ \\
\hline JEFE DE HOGAR & $4.9472 \%$ \\
\hline NIVEL EDUCATIVO & $3.3661 \%$ \\
\hline TASA DE DESEMPLEO-HOGAR & $9.0843 \%$ \\
\hline INGRESOS LABORALES & $0.000000873 \%$ \\
\hline REGIMEN SUBSIDIADO & $3.5619 \%$ \\
\hline PAREJA & $1.9475 \%$ \\
\hline
\end{tabular}

Fuente: Micro datos anonimizados de la Gran Encuesta Integrada De Hogares (GEIH), DANE. Cálculos Propios

La variable Sexo presenta un aumento en la probabilidad de participar en el mercado laboral de $21.5 \%$, lo que indica que ser hombre aumenta la probabilidad de pertenecer a la Población Económicamente Activa (PEA). De la misma manera, la variable Edad muestra un incremento en la probabilidad de la variable regresada de $0.3 \%$. Los anteriores datos son regulares en diferentes estudios científicos Sosa, María y Rosa Román encontraron una relación semejante en cuanto a la distribución total del tiempo por sexo en México, donde los hombres dedican casi el doble de tiempo respecto a las mujeres en trabajo remunerado (28.9 horas Vs 17.5 horas), no obstante también evidenció que las mujeres dedican casi tres veces más que los hombres al trabajo doméstico no remunerado (20.4 horas Vs 5.8 horas). (Sosa \& Rosa, 2015)

Igualmente, la variable Nivel Educativo presenta un aumento de $3.3 \%$ en la probabilidad de ser parte de la Población Económicamente Activa (PEA). El nivel educativo en el modelo, se refiere al último título otorgado a la persona, lo que significa que un título educativo adicional aumenta la probabilidad de participar laboralmente en el municipio de San José de Cúcuta y su Área Metropolitana.

La Tasa de Desempleo del Hogar también presenta un impacto positivo en la probabilidad de participar en el mercado laboral del 9.0\%, mostrando que aumentos en los desempleados del hogar son favorables en la decisión de participar. Así mismo, La Jefatura del Hogar incrementa en un $4.9 \%$ la probabilidad de participar laboralmente en el municipio.

Del mismo modo, aumentos en los ingresos laborales del individuo permiten un incremento del $8.73 * 10-07 \%$ en la probabilidad de ser parte de la Población Económicamente Activa del municipio. Pertenecer al Régimen Subsidiado y Tener pareja también presenta incrementos en la probabilidad de participar laboralmente con un porcentaje de $3.5 \%$ y $1.9 \%$ respectivamente.

En síntesis, todas las variables utilizadas en el modelo presentan impactos positivos en la probabilidad de participar en el mercado laboral del municipio de San José de Cúcuta y su Área Metropolitana; donde las variables Sexo y Tasa de Desempleo del Hogar presentan los mayores valores en el aumento de la probabilidad, y las variables Ingresos Laborales y Edad presentan los valores más bajos. Estos resultados son similares a los obtenidos en los trabajos anteriormente 
mencionados elaborados para Colombia, Cali e lbagué.

Para mejores interpretaciones, se realiza un ejercicio de simulación donde se muestran los cambios en la probabilidad cuando se presentan una o más de las características socioeconómicas del individuo y de su entorno, expresadas en cada una de las variables estudiadas. Por ejemplo, un hombre jefe de hogar aumenta la probabilidad de participar en el mercado laboral en un $26.4 \%$. Si adicionalmente posee pareja, pertenece al régimen subsidiado $y$ existen desempleados en su hogar, aumenta la probabilidad de participar laboralmente a $41.0 \%$.

Con el fin de determinar simulaciones para el caso de la mujer (Tabla 3), se halló la predicción de probabilidad de la variable sexo, donde 1 significa mujer y 0 hombre. El resultado indicó que ser mujer disminuye la probabilidad de pertenecer a la población económicamente activa en un 21,5\%; sin embargo, si la mujer es jefe de hogar su impacto negativo en la probabilidad de participar laboralmente se reduce a $-16,6 \%$; si adicionalmente hay existencia de desempleados en el hogar, la probabilidad de participar en el mercado laboral será de $-7,5 \%$; si además, la mujer posee nivel educativo y régimen subsidiado su probabilidad solo disminuirá en un $-0,5 \%$ y, si al mismo tiempo posee pareja, la probabilidad presentará un impacto positivo del 1,3\%. Mostrando, una mayor influencia positiva de las condiciones socioeconómicas en las mujeres.

Tabla $N^{\circ} 3$ Cálculo de probabilidades para la población femenina

\begin{tabular}{|l|l|}
\hline \multicolumn{1}{|c|}{ VARIABLE } & PROBABILIDAD \\
\hline SEXO & $-21.5526 \%$ \\
\hline EDAD & $0.3880 \%$ \\
\hline JEFE DE HOGAR & $4.9472 \%$ \\
\hline NIVEL EDUCATIVO & $3.3661 \%$ \\
\hline TASA DE DESEMPLEO-HOGAR & $9.0843 \%$ \\
\hline INGRESOS LABORALES & $0.000000873 \%$ \\
\hline REGIMEN SUBSIDIADO & $3.5619 \%$ \\
\hline PAREJA & $1.9475 \%$ \\
\hline
\end{tabular}

Fuente: Micro datos anonimizados de la Gran
Encuesta Integrada De Hogares (GEIH), DANE. Cálculos Propios

\section{Conclusiones}

El análisis descriptivo muestra un panorama particular para la ciudad de Cúcuta y su Área metropolitana, los bajos niveles de educación superior y altos niveles en básica primaria, sumados a alta cobertura del régimen subsidiado y población sin ningún tipo de seguridad social; reflejan el ausentismo del empleo formal en la ciudad.

Por otro lado, los resultados que arrojó el ejercicio econométrico permiten aceptar el modelo microeconómico Ocio-Consumo para explicar la decisión de participar en el mercado laboral, reconociendo las condiciones socioeconómicas de los individuos como núcleo fundamental de la oferta laboral.

El primer hallazgo del modelo se da en el intercepto, el cual presenta un efecto negativo. Indicando, que tanto hombres como mujeres son poco propensos a participar laboralmente en el municipio de San José de Cúcuta y su Área Metropolitana, cuando permanecen contantes las demás variables. Los signos de los coeficientes de las variables fueron positivos, lo que permite afirmar que las condiciones socioeconómicas afectan la decisión del individuo.

En el cálculo de las probabilidades se observa que las variables sexo, tasa de desempleo del hogar y jefatura del hogar, son las que mayor efecto positivo tienen en la probabilidad de participar laboralmente y los variables ingresos laborales, edad y pareja las que menor efecto presentan.

Para realizar comparaciones entre hombres y mujeres, se cambió el efecto en la variable sexo donde 1 significó mujer y 0 hombre. Y se encontró que en las mujeres se disminuye la probabilidad de participar laboralmente en 21,5\%; sin embargo, cuando se tienen en cuenta los resultados de las 
demás variables, la probabilidad de participar de la mujer se vuelve positiva.

\section{Referencias}

Aldana, D., \& Arango, L. E. (2007). Participación laboral en Ibagué. Banco de la república, Bogotá. Obtenido de http://repositorio.banrep.gov.co/bitstrea $\mathrm{m} /$ handle/20.500.12134/5457/be_439.pdf ?sequence $=1$ \&isAllowed $=\mathrm{y}$

Becker, G. (1965). A Theory of the Allocation of Time. The Economic Journal, 493-517. Obtenido de http://links.jstor.org/sici?sici=00130133\%28196509\%2975\%3A299\%3C493\% 3АATOTAO\%3E2.0.CO\%3B2-N

Becker, G. (1981). A treatise on the family. Massachussetts: Harvard University Press.

Becker, G. S. (1977). Teoría económica. (F. D. (FCE), Ed.) México, México: FONDO DE CULTURA ECONÓMICA (FCE).

Castellar, C., \& Uribe, J. I. (2001). Determinantes de la Participación en el Mercado de Trabajo del Área Metropolitana de Cali en Diciembre de 1998. Cali, Colombia. Obtenido de http://cms.univalle.edu.co/socioeconomia /media/ckfinder/files/DOCUMENTO\%20D E\%20TRABAJO\%20CIDSE\%20N\%C2\%B0\% 2056.pdf

Castiblanco, M. (2018). Emprendimiento informal y género: una caracterización de los vendedores ambulantes en Bogotá. Sociedad y Economía(34), 211-228.

Garavito, C. (2017). Oferta de trabajo del hogar remunerado en el Perú: 2007- 2014. cuadernos de economía, 265-299. doi:doi: 10.15446/cuad.econ.v36n72.57471
Killingsworth, M. r., \& Heckman, J. J. (1986). Female labor supply: a survey. En O. C. Ashenfelter, R. Layard, \& O. A. Layard (Ed.), Handbook of Labor Economics (Vol. I, págs. 103-204). North Holland: Elsevier science.

doi:https://doi.org/10.1016/S1573-

4463(86)01005-2

Pencavel, J. (1986). Labor supply of men: A survey. En O. C. Layard, Handbook of Labor Economics (Vol. I, págs. 3-103). doi:https://doi.org/10.1016/S15734463(86)01004-0

Posada, C. E., \& Arango, L. E. (2002). La participación laboral en Colombia. Banco de la República. Bogotá: Borradores de Economía. Obtenido de http://www.banrep.gov.co/es/borrador217

Posada, C. E., Arango, L. E., \& Charry, A. (2006). La participación laboral en Colombia según la nueva encuesta: Banco de la República, Bogotá. Obtenido de http://www.banrep.org/docum/ftp/borra2 50.pdf

Ribero, R., \& Meza, C. (1997). Determinantes de la Participación laboral de hombres y mujeres en Colombia en 1976-1995. Bogotá: DNP. Obtenido de https://colaboracion.dnp.gov.co/CDT/Estu dios\%20Econmicos/62.pdf

Santamaría, M., \& Rojas Delgadillo, N. (2001). La participación laboral: ¿qué ha pasado y qué podemos esperar. Departamento nacional de planeación, Bogotá. Obtenido de

https://colaboracion.dnp.gov.co/CDT/Estu dios\%20Econmicos/62.pdf

Sosa, M., \& Rosa, R. (2015). Participación y tiempo en actividades cotidianas de hombres y 
mujeres vinculados al mercado laboral en México. Sociedad y Economía(29), 63-89.

Tomaselli, A. (abril de 2017). Caracterización de la participación laboral en Chile. Series de la CEPAL(223), 1-57. Obtenido de https://www.cepal.org/es/publicaciones/4 1132-caracterizacion-la-participacionlaboral-chile

Uribe, J. I., Ortiz, C. H., \& Correa, J. B. (2006). ¿Cómo deciden los individuos en el mercado laboral? modelos y estimaciones para Colombia. Lecturas de economía(64), 59-89. Obtenido de http://aprendeenlinea.udea.edu.co/revista s/index.php/lecturasdeeconomia/article/vi ew/2650/2110 